\title{
Future work
}

No further aeromagnetic flights have been planned for the next couple of years but the follow-up ground work will start in 1977. Already in 1975-1976 a number of localities around Søndre Strømfjord andQôrqut in Godthåbsfjord have been visited and susceptibility values have been obtained. In 1977 several key areas will be visited. Magnetic ground surveys will be carried out over selected anomalies with the purpose of determining the source of the aeromagnetic anomalies. Susceptibilities will be measured in the field, and a collection of oriented samples will be gathered for rock magnetic studies to provide necessary information for more detailed interpretation of the aeromagnetic data. The collected samples will probably also be used in palaeomagnetic studies.

\section{Acknowledgements}

The two-year aeromagnetic programme in West Greenland has been a team effort involving other than GGU personnel. The Electronics Department of the Danish Atomic Energy Commission's Research Establishment, Ris $\emptyset$, has been responsible for most of the instrumental developments. In the field, E. Mose Christiansen, E. Hansen and S. Frost have almost constantly kept the instrumental system at peak performance under the often difficult arctic conditions. V. Søndergård, C. Marcussen, E. Nielsen, from University of Århus, and E. Hansen from GGU ably assisted in the base camp as well as under measuring flights. The pilots from Vængir Airtransport Co., Iceland, - T. Magnusson, B. Hjaltason, J. Valdimarson and A. Christiansen - carried out their work in a highly effective way and usually with great enthusiasm. Flight mechanic L. Atlason has competently ensured the maximum flying possibilities by carrying out the servicing of the aircraft at night.

\section{Reference}

Thorning, L. 1976: Aeromagnetic surveys in southern and central West Greenland between $63^{\circ}$ and $71^{\circ}$ N. Rapp. Grønlands geol. Unders. 80, 61-65.

\section{A brief description of the computer programs used by GGU in the treatment of aeromagnetic data}

\section{Leif Thorning}

Computer programs for the treatment of aeromagnetic data have been developed parallel to GGU's acquisition and compilation of basic aeromagnetic data. The first data were collected in East Greenland in 1974 (Larsen, 1975), followed in 1975 and 1976 by the systematic regional survey of parts of the west coast (Thorning, 1976; this report). The first programs of the system had been designed by the end of 1974 and since then there has been a 
gradual increase in the number of programs as well as in their effectiveness. The programs have been developed by K. Frellesvig, E. Kirsbo and P. Rasmussen of Geokon ApS in close cooperation with $\mathrm{H}$. C. Larsen and the writer from GGU, with additional support from GGU's computer group.

This note gives a status of the program system at the end of 1976 and will supply an easy reference for later publications on the aeromagnetic data. In the following sections a brief description will be given of the hardware available to GGU and the individual programs and their interrelationship.

\section{Hardware}

For aeromagnetic computational work facilities of the Regional Computing Center of Copenhagen University (RECKU, Kofoed \& Larsen, 1976) are used, although for some minor tasks the in-house facilities at GGU are utilised. These are centered around a PDP 11 minicomputer and consist of 4 discs, fast paper tape reader, paper tape puncher, three terminals one of which is a graphic-alfanumeric screen (Tectronix 4014-1) with attached graphic tablet (digitiser). The system is controlled through the executive system RSX11-M, which presently allows three simultaneous users of the system. This figuration is planned to be expanded in the very near future by a magnetic tape station and a plotter with enlargement of the central minicomputer. The RECKU system is an UNIVAC 1110 system with primary and extended storage and other facilities normal for a large computing center supporting both batch and demand processing. The above mentioned terminals also serve the connection to RECKU via 110, 300 or 1200 band transmission lines. Future plans include a possible closer merging of the two systems.

\section{Software}

In the design and production of the programs comprising the AM system care has been taken to make the system flexible and to make it easy to add new programs at any point in the system. Fig. 12 shows schematically the main programs of the AM system. Most of the programs have been written in FORTRAN V or IV; only some specialised routines performing certain machine dependant operations have been written in ASSEMBLER or COBOL. Some of the programs have two versions for use on RECKU or the minicomputer, respectively. A given program has been designed for either batch processing or demand processing, depending on the sort of operation it is meant for. Some programs will perform both ways by means of an option on the execute statement - enabling the user to do trial runs on small amounts of data before the final runs take place.

The programs are built up around the data base. A number of administrative programs performing checks, sorting and ordering of data have not been included on the diagram for reasons of clarity. The programs on the upper part of the diagram all deal with the preparation of data and the input of data to the data base, the lower part shows the programs dealing with the retrieval of data and with the interpretation and presentation of results. At any point in the system intermediate results can be stored in mass storage files or on tape (not shown on diagram) and new programs can be attached to the system as long as the input format corresponds to the format of the relevant intermediate file. Most programs provide graphical 
Fig. 12. Schematic representation of the computer program system used by the Survey for the treatment of aeromagnetic data. For full description see text.

AIRBORNE

U.

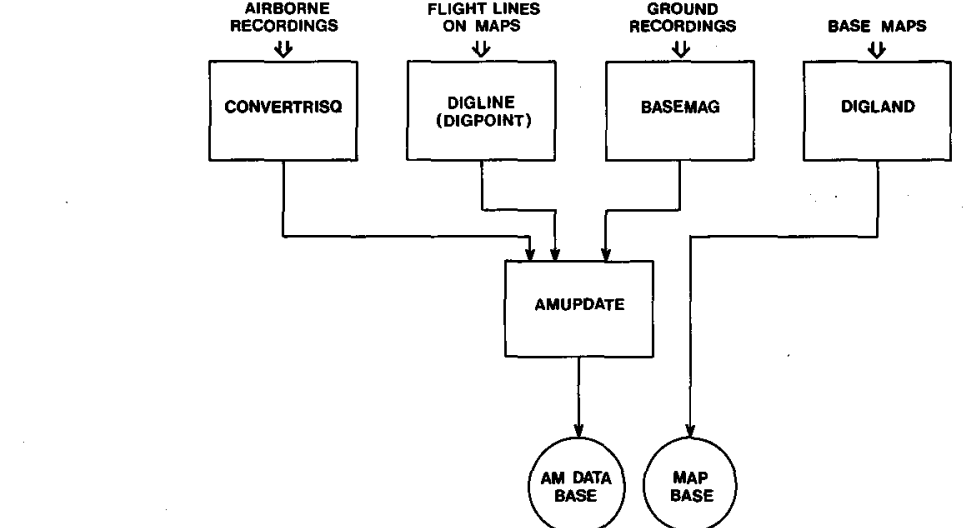

GRF

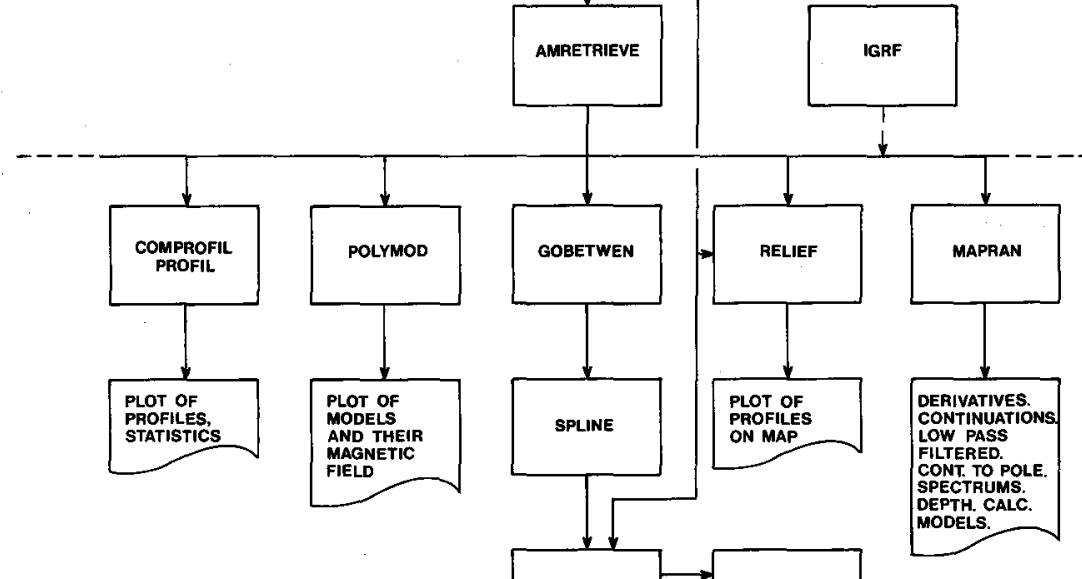

AMCONTOURS

HNDRSN

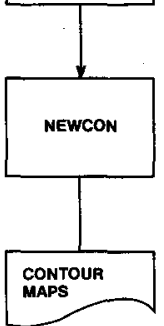

outputs (Tektronix screen or CalComp plotter) as well as the results used in further processing. The AM system is continuously being updated and optimised, and new programs are being added. 


\section{The AM data base}

All aeromagnetic data are organised in a data base making it easy to extract any combination of data. Extraction of data from the base is handled by AMRETRIEVE; input to the base is handled by AMUPDATE. For security reasons the system always maintains two versions of the data base, one containing all updatings and one containing all updatings but the last.

During one summer aeromagnetic surveys may be carried out over several regions and with different survey parameters. Each such survey area is labelled with two letters and the flight lines within the area are given a number. In this way all profiles are unambiguously defined. Each year has its own data base, and the data are subdivided according to survey area and map. Special files in the data base contain all necessary relations between profiles, maps and survey areas.

The position of profiles on a map is given in coordinates referring to a rectangular coordinate system, defined by geographical coordinates of the origo and with the $y$ axis pointing due north. In the future this may be rearranged so that a point can be refered to directly by its geographical coordinates.

For each measurement with the airborne equipment the data base contains the following information:

1. Profile number

2. Map identification

3. $(x, y)$ coordinates

4. Date

5. Time

6. Radio altitude
7. Barometric altitude

8. Airborne total field measurement

9. Base station measurement

10. Corrected total field value

11. Point qualifier (plotted or interpolated point)

\section{CONVERTRISQ}

On aeromagnetic measuring flights the data are recorded digitally on paper tape (Thorning, 1976) and these tapes are first treated at the Electronics Department of the Danish Atomic Energy Commission's Research Establishment, Risø. This department is responsible for much of the instrumental development in the aeromagnetic/radiometric projects undertaken by GGU. GGU receives both the radiometric and the magnetic data on magnetic tape in sequential order. The program CONVERTRISQ sorts out the magnetic data with relevant additional parameters and produces a magnetic tape with this data in a format suitable for further treatment on the installations of RECKU. At the same time it is possible to carry out corrections of data and time, if these happen to have been erroneously entered, and a few other simple corrections can be made.

\section{DIGLINE, DIGLAND and DIGPOINT}

Positions along flight paths are recorded with a $35 \mathrm{~mm}$ camera at regular intervals along the profile. These positions are plotted on stable base maps, and the profiles are then digitised using the program DIGLINE. This program enables the user to utilise the minicomputer system with the Tektronix screen and graphic tablet as an on-line digitiser. Additional 
parameters (e.g. time) and commands to the program are entered via the keyboard. The data are collected in a file, which at any time can be inspected and, if necessary, corrected in a number of ways. When the data are found satisfactory a paper tape is punched with the data in a format suitable as input to RECKU. More direct transfer to RECKU will be possible shortly. DIGLINE is presently being extended to include a routine enabling the user to plot the digitised map with flight lines on the screen for fast visual control. On RECKU the data undergoes certain coordinate transformations and is brought on a format suitable for input to AMUPDATE. In principle DIGLAND works in the same way, but instead of flight lines it deals with the map as such, i.e. coastlines, outlines of lakes, ice-covered regions etc. This information is not included in the AM data base, but kept on a seperate magnetic tape file. DIGPOINT extracts - via the graphic tablet - coordinates of points on a map and produces a table with the geographical coordinates and corresponding coordinates referring to the coordinate system of the map. This information is useful in later stages of the work.

\section{BASEMAG}

This is actually a number of modules used for the treatment of the temporal variations of the geomagnetic field as recorded digitally at the base station in the field. As a result of this treatment, which takes place partly at RECKU and partly at GGU, a number of files with all relevant information for the data base are produced in a format suitable for the input program AMUPDATE. At the same time it is possible to calculate certain statistical parameters and to obtain a measure of the geomagnetic activity in user defined periods of time.

\section{AMUPDATE}

This program initiates a data base for a given year and provides the facilities for all future updating of the base. The program is safeguarded making it impossible to damage the base by accident. By using different commands to the program the user can (1) merge raw data from the flight recordings (CONVERTRISQ) with position data (DIGLINE), (2) update the data base with ground station recordings (BASEMAG), (3) update with barometric altitude if this was not included in the original digital flight recordings, (4) correct magnetic total field values if for some reason the digital recording system has failed - this operation utilises the analogue recording, (5) delete profiles. The time interval between successive records is determined by the setting of the sampling rate of the airborne equipment.

\section{AMRETRIEVE}

This program makes it possible to extract data from the AM data base in almost any combination desired. The data retrieved are written in a file in well defined formats. To use the data as input to a program the input routines of that program only need correspondingly read statements. This makes it easy to adapt new programs to the system. Data can be retrieved on the basis of profiles or on the basis of maps up to any number of profiles or maps. When extracting the data from several maps in one operation the user can define a master coordinate system and the position of all profiles will then be transformed into coordinates of the master system. The user can also define the scale of the output maps. A certain 
combination of data is obtained by using a corresponding combination of options on the execute statement. Some of the parameters (3)-(11) will then be written in a file ordered firstly according to maps and secondly to profiles.

\section{GOBETWEEN}

For certain purposes the above mentioned arrangement of the data is not practical. GOBETWEEN can arrange the data in a more convenient way. With this program, the user can obtain data within a rectangular area and dismiss data outside this area. At the same time the different pieces of profiles found on different maps are put together and for each profile a new parameter is defined. All profiles within the area are arranged so that this parameter is measured from the same end of the profiles. The output from GOBETWEEN is again written in a file, which can be addressed from any relevant program.

\section{SPLINE}

This is a program taking its input from the above mentioned program. In many areas the number of measurements exceeds the number necessary for defining the actual variations recorded. SPLINE will take any number of profiles and for each profile the following operation will be performed. The original data points will be approximated by a cubic spline function. The accuracy of fit of this spline function is given by the user as the maximum deviation acceptable at any point. The program will then, if possible, determine the minimum number of points necessary for this demand and will remember the position of these points on the map. Inherent in this program is also the possibility of smoothing the data to a certain degree. SPLINE can be used interactively in a first assessment of new data or it can be operated in batch, i.e. processing a whole lot of profiles using the same limits for all the profiles. In the first case it provides the user with plots on the Tektronix screen for immediate evaluation, and if required, a possibility of obtaining a permanent CalComp plot. In batch processing standard plots are automatically produced. The thinning of data performed by SPLINE also decreases the need for core memory when using AMCONTOURS.

\section{AMCONTOURS}

This is major program of the Am system. It is a very versatile program providing a number of useful facilities for examination and representation of data. There are two main modules in the program, one calculating at regular intervals the values of the field to be contoured and the other performing the calculations associated with the actual plotting of the contours. A number of alternative outputs are also available. The input to the program is supplied by SPLINE. Interpolation to a grid is carried out by means of a method similar to that of Bhattacharyya (1969) using linear bicubic splines in two orthogonal directions. However, the improvement is offered that the profiles are not approximated by straight lines determined by least squares, but used directly. This eliminates errors caused by the interpolation of the field to straight lines. The user can define the direction and cell-size of the grid. Once the values of the field are known in all grid corners, a two-dimensional spline is used for interpolation of the field to the subgrid necessary for drawing the contours. There is a wide 
choice of possible contours. Special routines makes it possible to outline areas under contouring by a polygon. The program can be operated both in batch and demand. The latter is used on smaller amounts of data in preliminary analysis of the data or as a quick method of getting a good working knowledge of the data. Apart from actual contour maps a number of other possible outputs exist. The values of the field can be extracted in a user defined grid or along an arbitrary profile. Such outputs can either be plotted or written in a file. In the course of processing performed by AMCONTOURS various intermediate results of the compilation can be saved. A main exit from the program is a file with all information on the position of contours, extremum points and borders of the area. This file is input to NEWCON.

\section{NEWCON}

This program applies cosmetics to the contours produced by AMCONTOURS according to user requirements and produces a finished contour map with thinning of contours in high gradient areas, labelling of contours, maximum and minimum points and with a suitable frame of the map, It also offers a possibility of changing symbols of contours and using three different colours on the map. The digitised maps (DIGLAND) can be superimposed on the contour map.

\section{RELIEF}

This program plots the variations of the aeromagnetic field on a map along the true position of the profiles. It is used to present data unsuitable for contouring such as single profiles, and to plot closely spaced profiles so as to get a effect similar to that of 'stacking' profiles. The information obtained by DIGLAND can be superimposed on the plot produced by RELIEF.

\section{HNDRSN}

The program AMCONTOURS can produce gridded values of the magnetic field. HNDRSN takes these as input and calculates upward or downward continued field or derivatives of the field according to the formulas of Henderson (1960). The program is that of Rudman \& Blakely (1975) implemented at RECKU with some minor alterations. Besides the printer output described in Rudman \& Blakely (1975), it can also deliver output to a file making it possible to use parts of AMCONTOURS to produce contour maps of the transformed field.

\section{PROFIL and COMPROFIL}

These programs are used for the presentation of single profiles with time as one axis. PROFIL plots one parameter (a magnetic field value) and COMPROFIL plots any combination of parameters and enables the user to compare for instance ground clearance with total field, or airborne measurements with base measurements. For comparisons on a quantitative basis COMPROFIL can also produce files with relevant data for use in standard statistical programs of RECKU. 


\section{POLYMOD}

This is a collection of programs dealing with the problem of assigning a geological model to a given magnetic field, working in two or three dimensions. It includes models with polygonal cross-sections, and models to be composed of 'building bricks'. Most of these programs are interfaced to MAPRAN.

\section{IGRF}

The aeromagnetic data from Greenland will be related to the International Geomagnetic Reference Field. The program IGRF calculates the various components of this field. The user may apply either the 1965 or 1976 coefficients. The core of the program is the routine of Barraclough \& Malin (1971). Another slightly modified version of this routine is included in the program to achieve reference fields in which account has been taken of the secular acceleration (Barraclough et al., 1975). The program can deliver the output as tables or as gridded values.

\section{MAPRAN}

This is again a system of programs designed for magnetic profile analysis. The first modules deal with the ordering of data along a profile, digitising of data at regular intervals and other data administrative tasks. The programs are all used interactively with the Tektronix screen as primary output media, and the CalComp plotter as secondary output media. Besides the actual aeromagnetic data supplied from AMRETRIEVE, input can be added to from the screen by cursor or from files. In this way it is possible, for instance, to define regional fields and calculate residuals. When a profile has been ordered and represented at regular intervals within the most suitable limits and perhaps gone through a regional/residual separation, a number of operations can be performed on the profile. Whichever operation is performed the results will immediately be displayed on the screen for visual check before it is accepted. The methods forming the basis of the program are similar to those described by Green (1972) and Nabighian $(1972,1974)$. By using the Fast Fourier Transform and operating in the frequency domain the following transformations can be calculated: $N$ th the derivative field, upward and downward continued field, low pass filtered field. The power spectrum can be calculated and used in various analyses, e.g. statistical depth determinations. Other modules use the concept of an analytic signal (Nabighian, 1972) to outline models and calculate depths. Transformation to the pole is also possible. MAPRAN is a powerful tool in magnetic profile analyses. It is used for routine interpretations and also in more experimental interpretational work.

\section{Concluding remarks}

The programs of the AM system are constantly being updated and optimised in order to keep up with the facilities available and in order to include new methods. Work is also in progress on the possibilities inherently given in some of the programs, for instance the SPLINE representation of data in AMCONTOURS. Some of the programs may be the subject of future papers with more detailed accounts of theory and tests. 


\section{References}

Barraclough, D. R. \& Malin, S. R. C. 1971: Synthesis of international geomagnetic reference field values. Rep. Inst. geol. Sci. 71, 1, 26 pp.

Barraclough, D. R., Harwood, J. M., Leaton, B. R. \& Malin, S. R. C. 1975: A model of the geomagnetic field at epoch 1975. Geophys. J. Roy. Astron. Soc. 43, 645-660.

Bhattacharyya, B. K. 1969: Bicubic spline interpolation as a method for treatment of potential field data. Geophysics 34, 402-423.

Green, A. G. 1972: Magnetic profile analysis. Geophys. J. Roy. Astron. Soc. 30, 393-403.

Henderson, R. G. 1960: A comprehensive system of automatic computation in magnetic and gravity interpretation. Geophysics 25, 569-585.

Kofoed, E. \& Larsen, I. A. 1976: Brugervejledning. RECKU Publ. No. 1, 70 pp. Copenhagen.

Larsen, H. C. 1975: Aeromagnetic investigations in East Greenland. Rapp. Grønlands geol. Unders. 75, 88-91.

Nabighian, M. N. 1972: The analytic signal of two-dimensional magnetic bodies with polygonal cross-section: its properties and use for automated anomaly interpretation. Geophysics 37, 507-517.

Nabighian, M. N. 1974: Additional comments on the analytic signal of two-dimensional bodies with polygonal crosssection. Geophysics 39, 85-92.

Rudman, A. J. \& Blakely, R. F. 1975: Fortran program for the upward and downward continuation and derivatives of potential fields. Occ. Pap. Ser. Indiana geol. Surv. 10, 23 pp.

Thorning, L. 1976: Aeromagnetic surveys in southern and central West Greenland between $63^{\circ}$ and $71^{\circ}$ N. Rapp. Grønlands geol. Unders. 80, 61-65.

\section{Investigations of Quaternary geology on the west coast of Disko, central West Greenland}

\section{Joakim Donner}

During summer 1976 the coasts of Nordfjord, Mellemfjord and Disko Fjord were investigated. All three fjords were outlets for large valley glaciers moving towards the outer western coast of Disko. Some of the till material transported by the glaciers came from the inner parts of the island, as seen for instance in Nordfjord, where the material contains gneiss from the outcrops some way up in Stordal, the valley NW of the head of Nordfjord. However, the drift in the fjords consists mostly of the local basalt. During the retreat of the glaciers lateral moraines and kame terraces were deposited on the slopes. In Nordfjord they are at about 100 $m$ above sea level and at about the same altitude in the inner parts of Disko Fjord. No clear end moraines connected with the lateral formations can be traced, but thick drift near the mouth of Mellemfjord, at Narssârssuk on the southern shore and at Kûggssuaq on the northern shore, may form remnants of an end moraine. Similar thick drift occurs also on the southern and northern shores of the innermost parts of Kangikerdlak in Disko Fjord and at the mouths of some smaller valleys. 